\begin{tabular}{c} 
International Journal of Engineering \& Technology, 7 (1.1) (2018) 706-709 \\
International Journal of Engineering \& Technology \\
SPC \\
Website: www.sciencepubco.com/index.php/IJET \\
Research paper \\
\hline
\end{tabular}

\title{
EMG extractor and blink detection for human health monitoring
}

\author{
Aanchal Jha ${ }^{1 *}$, M. Ganesh Lakshamana Kumar ${ }^{2}$

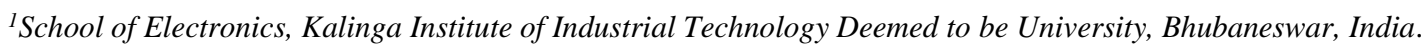 \\ ${ }^{2}$ Research Scholar, Indian Institute of Technology Hyderabad, Kandi, India. \\ *Corresponding author E-mail:12293aanchal@gmail.com
}

\begin{abstract}
In this paper we have proposed noise free EMG extractor for biomedical applications and also provide method for detecting blink signal. EMG signal is applied to preamplifier followed by Chebyshev filter and programmable gain amplifier further this processed EMG signal is applied to comparator to detect the blink. This topology is designed in UMC 180nm CMOS technology. Amplifier with gain of 81.155 $\mathrm{dB}$ and CMRR of $155.197 \mathrm{~dB}$ is designed. Preamplifier gain of $32.1244 \mathrm{~dB}$ with CMRR of $76.0743 \mathrm{~dB}$ which leads to common mode cancellation at priliminary stage. It also provide input referred noise ranges from 90 to $101.8636 \mu \mathrm{V} / \mathrm{sqrt}(\mathrm{Hz})$ to reduce the noise for overall system. 4th order Chebyshev filter provides filtering with slope of $-80 \mathrm{~dB} /$ decade with leads to reduce the unwanted signals. Filtered EMG signal is applied to programmable gain amplifier where gain ranges from 0 to $23 \mathrm{~dB}$.It consumes power of $0.3 \mu \mathrm{W}$ at $1 \mathrm{~V}$ supply voltage.
\end{abstract}

Keywords: Comparator, Current simulator, EMG extraction, functional electrical simulation (FES), programmable gain amplifier.

\section{Introduction}

In recent development biomedical signals acquisition plays important role in human health monitoring system. For this accurate biomedical signals are determined by filtering the unwanted signals and noises like power line noise, DC noise etc. Currently substitute to various input devices like keyborad, mouse [1],[2].which is known as human computer interface is in spotlight.

The study of bio potential signal like electroencephalogram (EEG), an electrooculogram (EOG), and an electromyogram (EMG) can be easily controlled by computer peripherals. In this biomedical signal, EMG signal plays important role since its intensity is easy to control hy HCI as it is generated from the motor units when the muscle contract. muscle. Diagnosys of the muscle can be done with the help of electromyography which is used in clinical and neural physiology. Proper working of muscle fibre can also be checked with the help of EMG, whose response in examined when external simulation is received or voluntary action is performed. Mostly it has been used in robotics and construction of active prosthetic elements. The main problem in implementing these application is that to record only the EMG signal ignoring the noises present. It should also be friendy to the users in terms of cost and portability.

In the laboratories of the state of art in significantly protected environment which should be insusceptible to noise. As other biomedical signal is also associated with the frequency band of EMG ,so it is very delicate and there is a high chance of interpreting of any biomedical signal to EMG signal .EMG signal works in the range of $50-150 \mathrm{~Hz}$ which is very close to the power line noise i.e.of $50 \mathrm{~Hz}$. So, effort in removing this noise may affect the EMG signal. This signal is also affected from the skin's surface condition. Different techniques have been developed to get rid of the problems realed to EMG signal[3]-[6].To achieve configurable gain[3] a variable gain amplifier (VGA) has been enrolled. Here gain can be controlled manually. So the adaptive control logic is preferred for adjusting the gain of VGA[4] automatically.

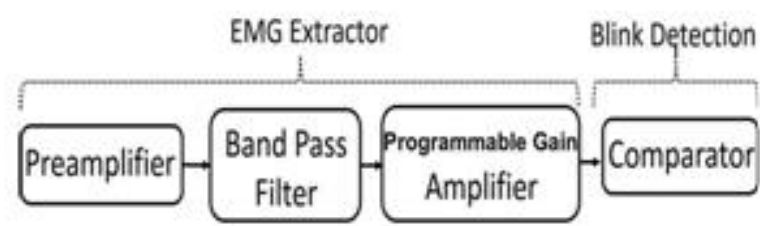

Fig. 1: Block diagram of EMG extractor

The key objective of this project is to extract the EMG signal for the patients. Table-1 shows the comparative analysis of previously done work [7-10] on the EMG extraction and the work which is performed by me in this paper. The paper is organized as follows:- Section II describes block diagram and section III is about the description of circuit. Section IV shows the simulation results and conclusion is stated in section $\mathrm{V}$.

\section{Block diagram}

Fig-1 shows block diagram of EMG extractor with blink detection which consists of preamplifier, band pass filter, programmable gain amplifier and comparator. Movement of muscle are captured and applied to preamplifier for amplification of EMG signal with noise. Preliminary amplifier filtered unwanted signals with slope of $-20 \mathrm{~dB} / \mathrm{decade}$. Further this amplified signal is passed through 
strong 4th order Chebyshev filter to filter unwanted signals by a factor more than-40dB. Programmable gain amplifier amplifies filtered EMG signal to milivolts such that further processing. Comparator com- pares EMG signal with threshold voltage of blink and provides blink detection result at the output.

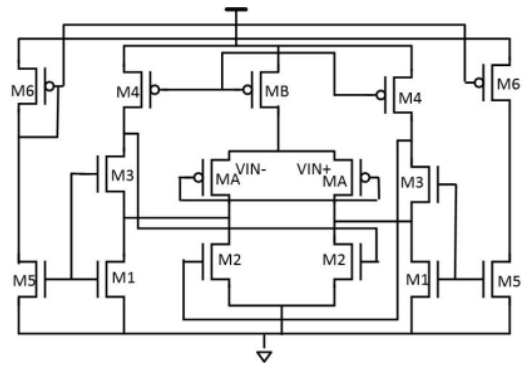

Fig. 2: Amplifier circuit

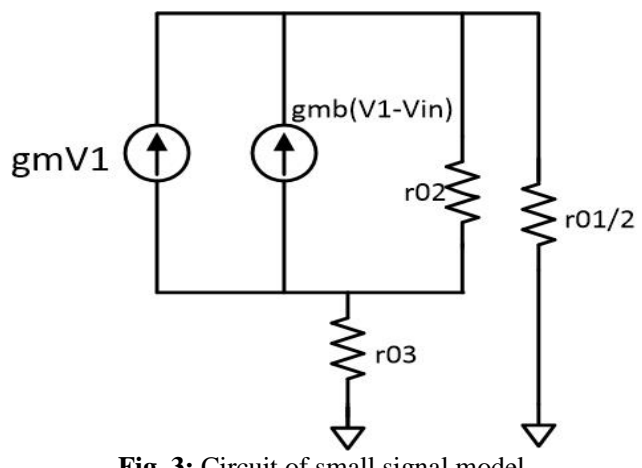

Fig. 3: Circuit of small signal model

\section{Circuit description}

\subsection{Amplifier design}

Fig-2 shows the transistor level realization of bulk driven input stage. Transistor with the same name are alike. Here input is given to the bulk of transistor $\mathrm{M}_{\mathrm{A}}$ and the path are joined to the ground so that suitable value of gate-source voltage is achieved. $\mathrm{I}_{\mathrm{B}}$ which is biasing current flows through the transistor $\mathrm{M}_{\mathrm{B}} . \mathrm{M}_{1}$, $\mathrm{M}_{3} \& \mathrm{M}_{5}$ act as low voltage current mirror. $\mathrm{I}_{4}$ flows through the transistor M4 to keep the mirror active. Partial positive feedback loop is performed so transistor $\mathrm{M}_{2}$ is connected which will not affect the frequency stability as $\mathrm{M}_{1}$ has smallest aspect ratio to $M_{2}$. Here differential to single ended conversion is done by folded cascode technique. Transistor $\mathrm{M}_{6}$ act as current mirror so that current can be copied keeping the output current constant.

\subsection{Preamplifier}

The signal is of $\mu \mathrm{V}$ range which is very small and we cannot apply any process to that signal so it needs to be preamplified by around 20 times so that common mode interference can be excluded. $\mathrm{Rf} * \mathrm{C} 2$ is the higher cut off frequency and gm of internal circuit CL will man- age lower cut off frequency, gain is provided by $\mathrm{C} 1 / \mathrm{C} 2$. Topology of preamplifier is shown in fig- 4 .

\subsection{Band pass filter}

It is a device which is used to pass the frequency of certain range and the frequency which lies outside its range is rejected. In this design we have used the band pass filter and it is Chebyshev Band

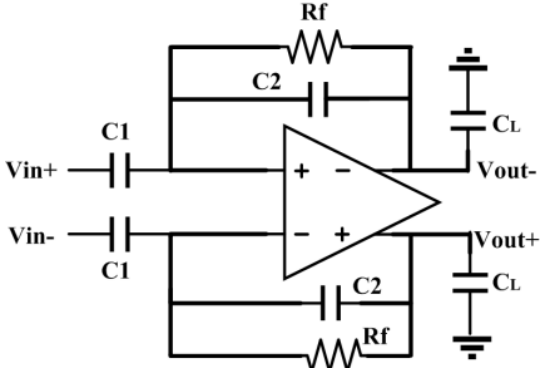

Fig. 4: Preamplifier circuit

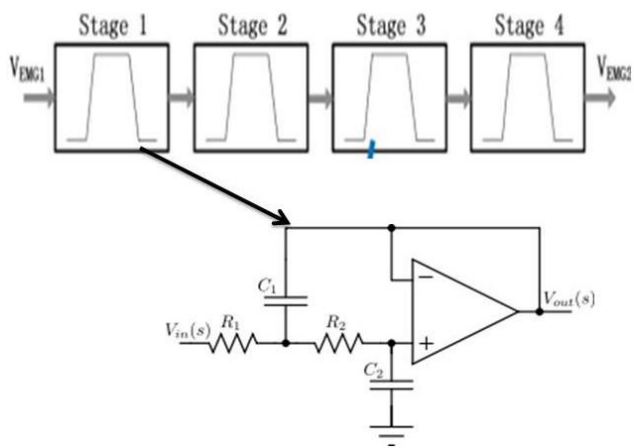

Fig. 5: Filter circuit

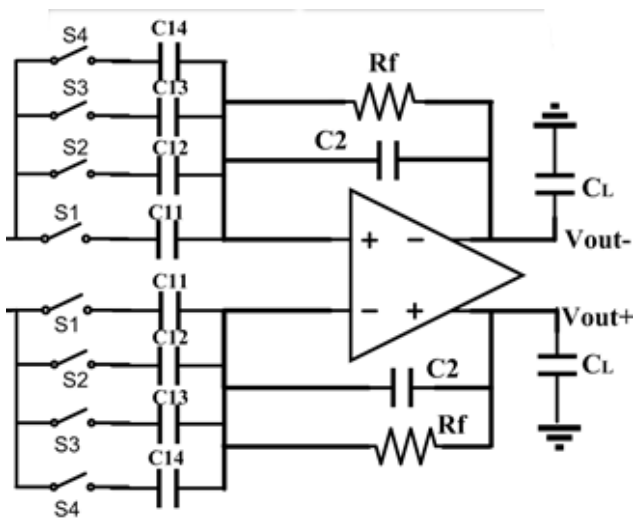

Fig. 6: Programmable Gain Amplifier

pass filter of order four. We are using Chebyshev Band Pass filter because the major advantage of this filter is that it allows ripple in the passband amplitude response. Equal ripple response filter is the another name by which Chebyshev filter is denoted and it also has a sharper transition band. With the Chebyshev band pass filter we get the needed frequency for the detection of blink through which signal can be amplified. If four stage of an all pole active filter is cascaded then execution can be done. As shown in fig-5 two capacitor whose values are taken as $10 \mathrm{nF}$ and $\mathrm{R} 1$ and $\mathrm{R} 2$ control the amplification factor and also four preamplifier are used for designing the filter.

\subsection{Programmable gain amplifier}

It is called as programmable gain amplifier because gain can be controlled by both analog and digital signals. Gain depends on the switch, and when all switch are on then gain $=(\mathrm{S} 1 \mathrm{C} 1+\mathrm{S} 2 \mathrm{C} 2+\mathrm{S} 3 \mathrm{C} 3+\mathrm{S} 4 \mathrm{C} 4) / \mathrm{Cf}$ as shown in figure 6.If switches are on then the corresponding capacitance supports for increase in gain. 


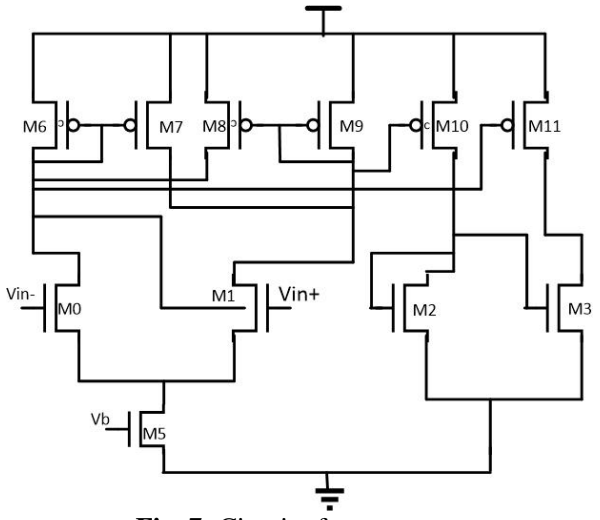

Fig. 7: Circuit of comparator

\subsection{Comparator}

Fig-4 shows the circuit diagram of comparator. Comparison of volt- ages or currents is done by a comparator and its output is a digital signal which states clearly which is larger. It consists of analog $\mathrm{i} / \mathrm{p}$ as Vin+,Vin- and digital $\mathrm{o} / \mathrm{p}$ as V0. High-gain differential amplifier is present in the comparator. Mainly it is used to measure and digitize analog signals, such as analog-to-digital converters.

\begin{tabular}{llcccc}
\multicolumn{7}{c}{ Table 1: Comparative Analysis of Amplifier } \\
\hline PARAMETER & THIS & {$[7]$} & {$[8]$} & {$[9]$} & {$[10]$} \\
& WORK & & & & \\
Supply voltage(v) & 1 & +0.9 & 1.8 & 1 & 1.8 \\
\hline CMOS & 180 & 180 & 180 & 180 & 180 \\
Gain $(\mathrm{dB})$ & 81.1579 & 80.24 & 60.9 & 58.25 & 74 \\
\hline GBW $(\mathrm{MHz})$ & - & 6.03 & 134.2 & 79.53 & 160 \\
\hline Phase & - & 73.8 & 70.6 & 61 & - \\
\hline CMRR & 155.197 & 145.09 & - & - & - \\
PSRR & - & 86.29 & - & - & - \\
\hline Slew rate $(\mathrm{V} / \mu \mathrm{s})$ & - & 3.67 & 94.1 & 2.3 & 26.6 \\
\hline Power $(\mu \mathrm{W})$ & 0.1 & 93.6 & 1440 & 67 & 362 \\
\hline $\mathrm{I}_{\text {bias }}(\mu \mathrm{A})$ & 1 & 11.05 & - & - & - \\
\hline THD for & - & -48.78 & - & - & - \\
$1.4 \mathrm{~V}_{\mathrm{pp}}(\mathrm{dB})$ & & 2.52 & - & - & - \\
\hline $\mathrm{I}_{\text {noise }}(\mu \mathrm{V} / \mathrm{sqrt} \mathrm{Hz})$ & 0.344 & 5 & 5.6 & 5 & 1.75 \\
\hline $\mathrm{C}_{\mathrm{L}}(\mathrm{pf})$ & 10 & - & 31.78 & 0.17 & 0.129 \\
\hline FOM & - & & & &
\end{tabular}

\section{Simulation results}

Design of EMG Extractor is done in UMC 180nm CMOS technology. It draws $0.3 \mu \mathrm{A}$ current with $1 \mathrm{~V}$ VDD. Fig-3 shows the small signal model of the amplifier circuit. Fig- 8 presents gain of the amplifier circuit which is $81.1579 \mathrm{~dB}$ and common mode rejection ratio is $155.197 \mathrm{~dB}$. Fig-9 describes about the input referred noise of the circuit at $270 \mathrm{~Hz}$ is 344.3128 $\mathrm{nV} / \mathrm{sqrt}(\mathrm{Hz})$ and at $470 \mathrm{~Hz}$ is $315.4379 \mathrm{nV} / \mathrm{sqrt}(\mathrm{Hz})$.In Fig-10 the gain of the preamplifier is

32.1244 dB.In Fig-11 the input referred noise at $270 \mathrm{~Hz}$ is $101.8636 \mu \mathrm{V} / \mathrm{sqrt}(\mathrm{Hz})$ and at $470 \mathrm{~Hz}$ is $90.32622 \mu \mathrm{V} / \mathrm{sqrt}(\mathrm{Hz})$ of preamplifier circuit.Fig-12 shows the plot of filter with slope $80 \mathrm{db} /$ decade. Fig-13 shows Programmable Gain is achieved from $0 \mathrm{~dB}$ to $23 \mathrm{~dB}$

@ $47 \mathrm{~Hz}$ to $78 \mathrm{~Hz}$.Digital output from processor is received as $0.6 \mathrm{~V}$

which is shown in fig-14.Layout design of amplifier is shown in

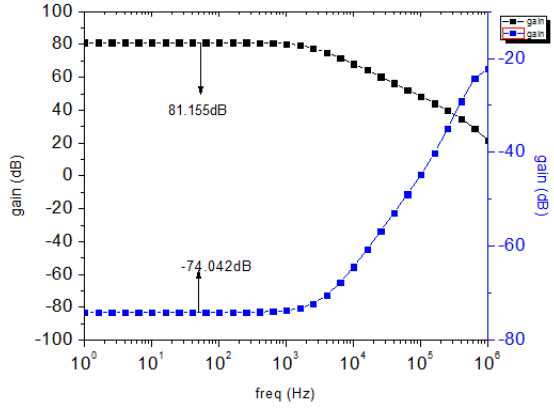

Fig. 8: Gain and CMRR plot of amplifier circuit

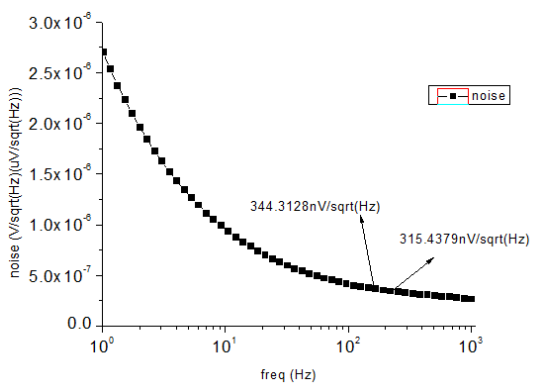

Fig. 9: Noise plot of amplifier circuit

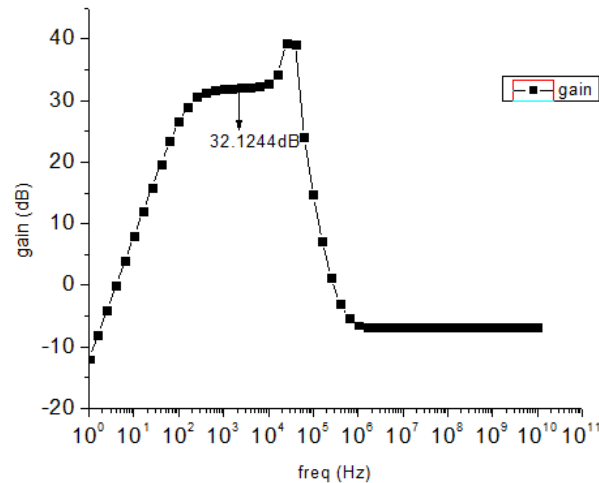

Fig. 10: Gain plot of preamplifier circuit

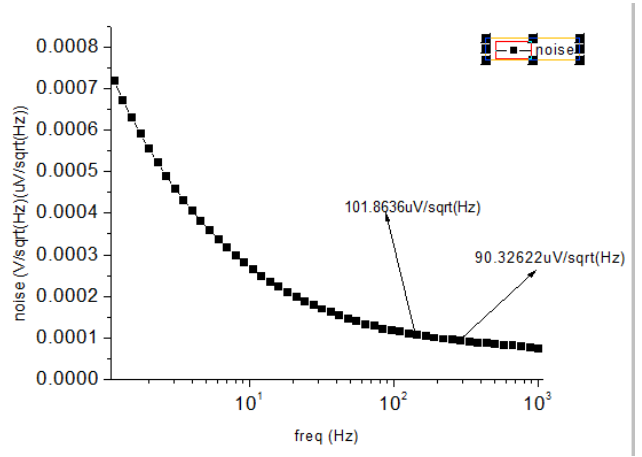

Fig. 11: Noise plot of preamplifier circuit

fig-15 having area $83.2 \mu \mathrm{W} \times 91.7 \mu \mathrm{W}$. Fig-16 shows the layout of preamplifier with area is $332.76 \mu \mathrm{W} \times 206.005 \mu \mathrm{W}$ 


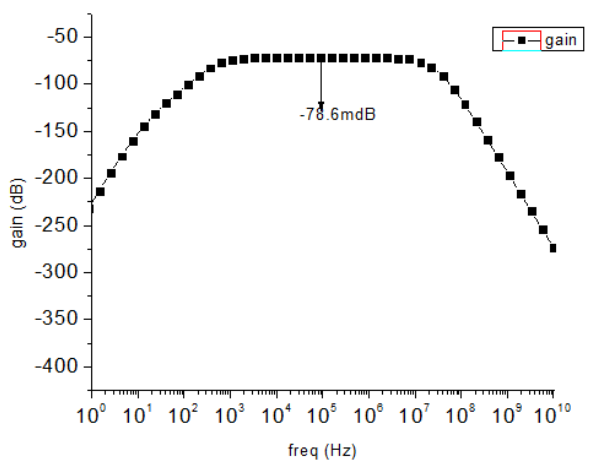

Fig. 12: Plot of filter

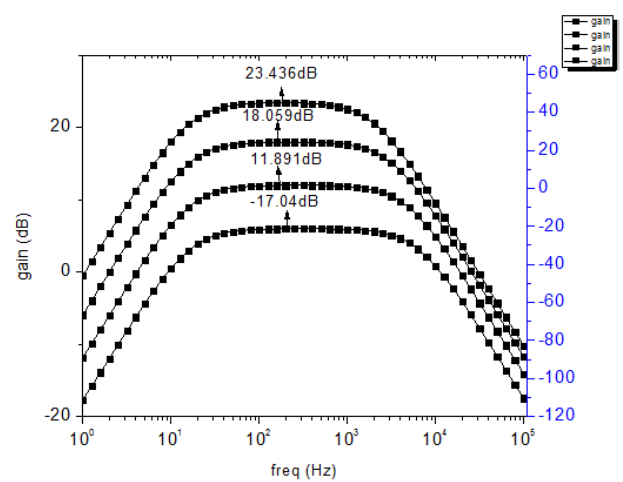

Fig. 13: Simulation result of programmable gain amplifier

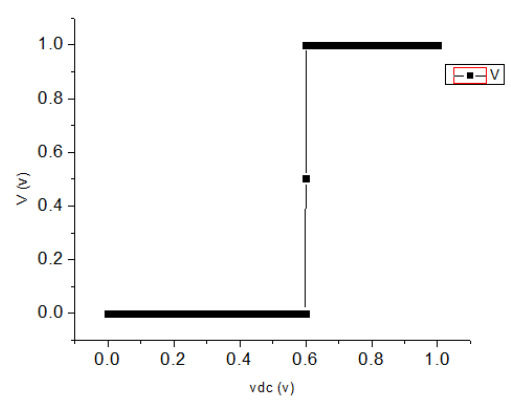

Fig. 14: Plot of comparator

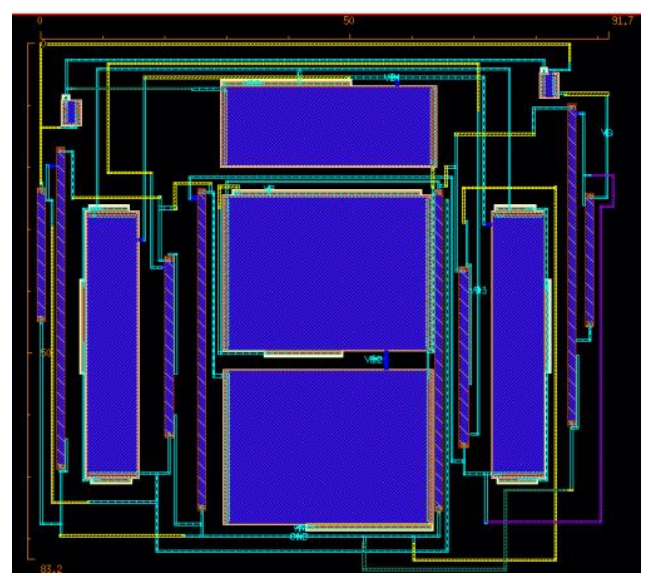

Fig. 15: Layout of amplifier

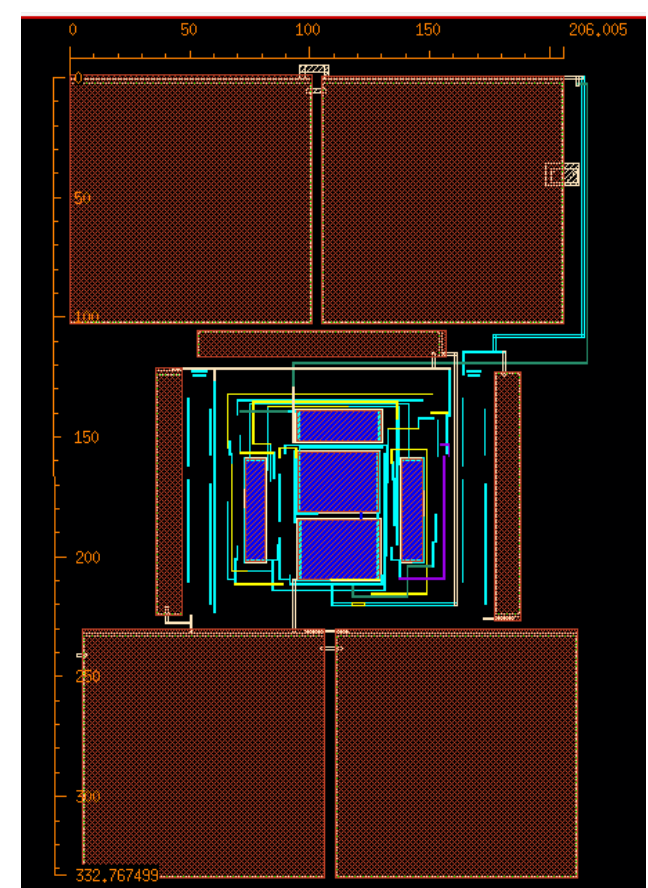

Fig.16: Layout of preamplifier

\section{Conclusion}

The proposed EMG extractor is low power of $0.3 \mu \mathrm{W}$ and low noise of $101.8636 \mu \mathrm{V} / \mathrm{sqrt}(\mathrm{Hz})$ in the operating frequency $270 \mathrm{~Hz}-470$ $\mathrm{Hz}$. It is also designed for EMG extractor without noise and unwanted signals. Simulation results shows that preamplifier provides high CMRR to cancel common mode signals, Chebyshev filters helps to remove unwanted signals .Blink detection is done by placing proper threshold voltage levels.

\section{References}

[1] Oonishi Y, Oh S \& Hori Y, "A new control method for power assisted wheelchair based on the surface myoelectric signal", IEEE Trans- actions on Industrial Electronics, Vol. 57, No. 9, (2010), pp.3191-3196.

[2] Artemiadis PK \& Kyriakopoulos KJ, "An EMG-based robot control scheme robust to time-varying EMG signal features", IEEE Trans-actions on Information Technology in Biomedicine, Vol.14, No.3, (2010), pp.582-588.

[3] Yazicioglu RF, Merken P, Puers R \& Van Hoof C, “A $60 \mu$ W 60 $\mathrm{nV} / \mathrm{sqrtHz}$ readout front-end for portable biopotential acquisition systems", IEEE Journal of Solid-State Circuits, Vol.42, No.5, (2007), pp.1100-1110.

[4] Li Q, Tan KHR, Hui TT \& Singh R, “A 1-V 36- $\mu$ W low-noise adaptive interface IC for portable biomedical applications", European Solid-State Circuits Conference, (2007), pp.288-291.

[5] Rieger R, "Variable-gain, low-noise amplification for sampling front ends", IEEE Transactions on Biomedical Circuits and Systems, Vol.5, No.3, (2011), pp.253-261.

[6] Hsu YP, Lin YT, Chen CH \& Lu SS, "A feed-forward automaticgain control amlifier for biomedical applications", Proceedings of Asia-Pacific Microwave Conference, 2007.

[7] Tripurari S \& Nipu K, "Nath Low-power, Folded Cascode Near Rail-to-Rail OTA for Moderate Frequency Signal Processing Innovations in Electronics", IEEE International Conference on Signal Processing and Communication (IESC), (2017).

[8] Assaad R \& Silva-Martinez J, The recycling folded cascode: a general enhancement of the folded Cascode amplifier", IEEE $J$ Solid-State Circuits, Vol. 44, (2009), pp.25352542.

[9] Liu A \& Yang H, Low voltage low power class-AB OTA with negative resistance load", IEEE Int. Conf. Commun., Circuits, Syst., Vol. 4, (2006), pp.22512254.

[10] Perez A, Nithin K, Bonizzoni E \& Maloberti F, "Slew-rate and gain enhancement in two stage operational amplifiers", IEEE Int.Symp. Circuits Syst., (2009), pp.24852488. 